\title{
Circulating $\mathrm{CX} 3 \mathrm{CR} 1^{+} \mathrm{CD} 163^{+} \mathrm{M} 2$ monocytes markedly elevated and correlated with cardiac markers in patients with acute myocardial infarction
}

\author{
Xia Shao ${ }^{1 \#}$, Boting Wu ${ }^{2 \#}$, Pu Chen ${ }^{3}$, Fanli Hua ${ }^{4}$, Luya Cheng ${ }^{1}$, Feng Li $^{1,4}$, Yanxia Zhan ${ }^{1}$, Chanjuan Liu ${ }^{1}$, \\ Lili Ji ${ }^{1}$, Zhihui Min ${ }^{5}$, Lihua Sun ${ }^{4}$, Yunfeng Cheng ${ }^{1,4,5,6}$, Hao Chen ${ }^{7}$ \\ ${ }^{1}$ Department of Hematology, ${ }^{2}$ Department of Transfusion Medicine, ${ }^{3}$ Department of Clinical Laboratory, Zhongshan Hospital, Fudan University, \\ Shanghai 200032, China; ${ }^{4}$ Department of Hematology, Zhongshan Hospital Qinpu Branch, Fudan University, Shanghai 201700, China; ${ }^{5}$ Institute \\ of Clinical Science, Zhongshan Hospital, Fudan University, Shanghai 200032, China; ${ }^{6}$ Center for Tumor Diagnosis \& Therapy, Jinshan Hospital, \\ Fudan University, Shanghai 201508, China; ${ }^{7}$ Department of Thoracic Surgery, Zhongshan Hospital Xuhui Branch, Fudan University, Shanghai \\ 200031, China \\ Contributions: (I) Conception and design: X Shao, B Wu, H Chen, Y Cheng; (II) Administrative support: Y Zhan; (III) Provision of study materials or \\ patients: X Shao, Y Zhan, B Wu; (IV) Collection and assembly of data: P Chen, Y Zhan, Z Min, C Liu, L Sun; (V) Data analysis and interpretation: \\ P Chen, Y Zhan, Z Min, C Liu, L Sun; (VI) Manuscript writing: All authors; (VII) Final approval of manuscript: All authors. \\ \#These authors contributed equally to this work. \\ Correspondence to: Hao Chen, MD, PhD. Department of Thoracic Surgery, Zhongshan Hospital Xuhui Branch, Fudan University, Shanghai 200031, \\ China. Email: h.chen@fudan.edu.cn; Yunfeng Cheng, MD, PhD. Department of Hematology, Zhongshan Hospital, Fudan University, Shanghai \\ 200032, China. Email: yfcheng@fudan.edu.cn.
}

Background: Vulnerable plaques have been generally recognized to play a role in the pathogenesis of acute myocardial infarction (AMI), however, the role of circulating CX3CR $1^{+} \mathrm{CD} 163^{+} \mathrm{M} 2$ monocytes has not been studied properly. We aim to evaluate the features of $\mathrm{CX} 3 \mathrm{CR} 1^{+} \mathrm{CD} 163^{+} \mathrm{M} 2$ monocytes and its relationship with cardiac specific markers in AMI patients.

Methods: The circulating M2 monocytes were identified in AMI patients $(n=35)$ and healthy controls (HCs, $\mathrm{n}=10$ ) by flow cytometry using two staining methods: $\mathrm{CD}^{+} 8^{+} \mathrm{CD} 163^{+}$(cytoplasmic staining) and $\mathrm{CX} 3 \mathrm{CR} 1^{+} \mathrm{CD} 163^{+}$(surface staining). CX3CR1 $1^{+}$monocytes were purified by magnetic cell sorting. The expression level of peroxisome proliferator-activated receptor $\gamma(\operatorname{PPAR} \gamma)$ and arginase-1 (Arg-1) were measured by real-time quantitative PCR and Western Blot in CX3CR $1^{+}$monocytes.

Results: Circulating M2 monocytes extremely expanded in AMI patients compared with $\mathrm{HCs}(\mathrm{P}<0.01)$. Positive linear correlation was confirmed between $\mathrm{CD} 68^{+} \mathrm{CD} 163^{+}$and $\mathrm{CX} 3 \mathrm{CR} 1^{+} \mathrm{CD} 163^{+}$cell populations in AMI patients $(\mathrm{r}=0.39, \mathrm{P}=0.02)$. The percentage of circulating $\mathrm{CX} 3 \mathrm{CR} 1^{+} \mathrm{CD} 163^{+} \mathrm{M} 2$ monocytes positively correlated with cardiac specific markers (cTNT, CK-MB) and acute phase markers (glucose, hs-CRP) (cTNT, $\mathrm{r}=0.63, \mathrm{P}<0.01$, CK-MB, r=0.54, $\mathrm{P}<0.01$, glucose, $\mathrm{r}=0.62, \mathrm{P}<0.01$, hs-CRP, $\mathrm{r}=0.58, \mathrm{P}<0.01)$. CX3CR1 ${ }^{+}$ monocytes in AMI patients expressed higher levels of PPAR $\gamma$ and Arg-1 than those in $\mathrm{HCs}(\mathrm{P}<0.01)$.

Conclusions: Circulating M2 monocytes increased in AMI patients and positively correlated with the elevation of both cardiac specific and acute phase markers. CX3CR $1^{+} \mathrm{CD} 163^{+} \mathrm{M} 2$ monocytes might have application value for the early diagnosis of AMI.

Keywords: Acute myocardial infarction (AMI); M2 monocytes; cardiac markers

Submitted Jan 07, 2020. Accepted for publication Mar 20, 2020.

doi: 10.21037/atm-20-383

View this article at: http://dx.doi.org/10.21037/atm-20-383 


\section{Introduction}

Acute myocardial infarction (AMI) is the direst form of coronary artery diseases, which primarily results from vulnerable atherosclerotic plaques (1). Its prompt and accurate diagnosis are critical for the initiation of effective evidence-based medical management. Cardiac troponin (cTn) is one of the diagnostic biomarkers for the evaluation of AMI. However, serial blood sampling is required for the diagnosis of non-ST-elevation myocardial infarction (2). The delayed rule-in increases mortality. Given the fact that nonspecific inflammation plays a critical pathogenic role in plaque disruption $(3,4)$ and macrophages are the primary source of inflammation effectors, the role of macrophages in atherosclerosis have enticed scientists to explore for decades.

Depending on the remarkable plasticity, macrophages can be polarized to either classical M1 macrophages or alternative M2 macrophages. M1 macrophages are pro-inflammatory macrophages exhibiting increased phagocytic activity while M2 macrophages mitigate over exuberant inflammation and play a vital role in promoting cardiac healing and remodeling process after myocardial infarction (5). Although M1 and M2 coexist in the atherosclerotic lesions, growing body of evidence have highlighted the central role of M1 in exuberant inflammation thus driving the destabilization of internal atheromatous plaques $(6,7)$. However, pathological intraplaque angiogenesis has recently been reported to contribute to the plaque destabilization (8). Neovessels in vulnerable plaques are characterized by discontinuous basement membrane, low number of tight junctions between the endothelial cells and poor pericyte coverage, which are highly susceptible to the leakage of circulating cell thus inducing intraplaque haemorrhage (9-11). Therefore, M2 macrophages might be of clinical importance as it could regulate intraplaque vessel sprouting and function in angiogenesis $(12,13)$. M2 macrophages have not been studied clearly in AMI patients.

CD163 and Chemokine-X3C-receptor-1 (CX3CR1) have already been generally recognized as the typical M2 markers. CX3CR $1^{+} \mathrm{CD} 163^{+}$monocytes were also precisely defined as M2 monocytes $(14,15)$. CX3CR $1^{+}$cells have been found markedly upregulated and facilitated inflammatory infiltration in atherosclerosis lesions (16). However, the presence of circulating $\mathrm{CX} 3 \mathrm{CR} 1^{+} \mathrm{CD} 163^{+} \mathrm{M} 2$ monocytes and their association with inflammation remains largely unknown in AMI.

Macrophages phenotype is heterogeneous, and transcription factors are important for its polarization by inducing epigenetic changes. Peroxisome proliferator-activated receptor $\gamma(\operatorname{PPAR} \gamma)$ held potent anti-inflammatory properties that could skew human monocytes towards M2 macrophages (17). Arginase-1 (Arg-1), predominantly expressed by M2 macrophages, could alter the magnitude and duration of the immune response (18). The present study aimed to study the correlation between circulating M2 monocytes and cardiac specific and acute-phase markers in AMI patients. We further looked into the function of circulating M2 monocytes by investigating the expression of PPAR $\gamma$ and Arg-1. These insights may provide glimpses into potential clinical significance of M2 monocytes in AMI.

\section{Methods}

\section{Patients and clinical parameters}

Thirty-five patients diagnosed with AMI scheduled for percutaneous coronary intervention (PCI) were enrolled in the study between April and July 2018. All patients met the international diagnosis criteria of AMI (1). Clinically unstable patients with cardiac arrest, cardiogenic shock or uncontrolled hypotension, as well as cases with stable angina pectoris, acute malignant disease, and chronic inflammatory diseases were excluded. Patient age, gender and the clinical parameters were recorded. Age matched controls consisted of ten healthy volunteers. The present study was conducted in accordance with the Declaration of Helsinki. The study protocol was approved by our Institutional Review Board, signed informed consent was obtained from all patients.

\section{Isolation of peripheral blood mononuclear cells}

EDTA-anticoagulated venous blood samples were immediately drawn from AMI patients before $\mathrm{PCI}$ and from healthy controls (HCs). PBMCs were isolated by Ficoll density-gradient centrifugation and resuspended in cell freezing medium (10\% DMSO in fetal bovine serum) at $5 \times 10^{5}$ cells $/ \mathrm{mL}$. The cell pellets were frozen at $-80^{\circ} \mathrm{C}$ and thawed for testing on separate occasions.

\section{Flow cytometry analysis}

Two methods were used to identify circulating M2 monocytes: intracellular staining methods $\left(\mathrm{CD} 68^{+} \mathrm{CD} 163^{+}\right)(19)$ and cell surface staining methods $\left(\mathrm{CX} 3 \mathrm{CR} 1^{+} \mathrm{CD} 163^{+}\right)(14,15)$. CD68 (Y1/82A; BD Biosciences, San Jose, CA, USA) and CD163 (GHI/61; BD Biosciences) were intracellularly stained after fixing 
with $4 \%$ paraformaldehyde and permeabilizing by FACS permeabilizing solution (BD PharMingen, San Diego, CA, USA). The cell-surface staining was performed to assess CX3CR1 (2A9-1; BioLegend, San Diego, CA, USA) and CD163 (GHI/61; BD Biosciences) expression, according to the manufactures' instructions. The PBMCs were gated by FSC $\times$ SSC (Figure S1). Flow analysis was performed on a FACS Aria II flow cytometer (BD Biosciences) and data were analyzed using FlowJo 7.6.1 (Tree Star, San Carlos, CA, USA) software.

\section{Magnetic cell separation of CX $3 C R 1^{+}$monocytes}

From the PBMCs, pure $\mathrm{CX} 3 \mathrm{CR} 1^{+}$monocytes were separated by positive selection, using CX3CR1 MicroBeads and a magnetic cell sorting (MACS) separator (Miltenyi Biotech, Bergisch Gladbach, Germany) according to the manufacturer's instruction. The purity of the cells after sorting was $>98 \%$ (Figure S2).

\section{Real-time quantitative PCR}

Total RNA was extracted from CX3CR1 ${ }^{+}$monocytes using TRIzol Reagent (Invitrogen, Carlsbad, USA) and converted into cDNA using PrimerScript RT Master Mix (Takara, Tokyo, Japan), then was performed using SYBR Premix Ex Taq (Takara, Tokyo, Japan) on an Applied Biosystems 7500 Fast Real-Time PCR System. Expression of GAPDH was used as internal reference. The following primer sequences were used: GAPDH forward: AACAGCCTCAAGATCATCAG, reverse: AGTCCTTCCACGATACCAA; PPAR $\gamma$ forward: ACCAAAGTGCAATCAAAGTGGA, reverse: ATGAGGGAGTTGGAAGGCTCT; Arg-1 forward: GTGGAAACTTGCATGGACAAC, reverse: AATCCTGGCACATCGGGAATC.

\section{Western blot}

CX3CR $1^{+}$monocytes were homogenized in ice-cold lysis and then were clarified by centrifugation $\left(12,000 \mathrm{~g}, 4^{\circ} \mathrm{C}\right.$, $30 \mathrm{~min})$. Equivalent protein $(20 \mu \mathrm{g})$ was subjected to a $10 \%$ sodium dodecyl sulfate-polyacrylamide gel and then transferred to polyvinylidene fluoride membranes (Millipore, Bedford, USA). Membranes were blocked for $1 \mathrm{~h}$ at room temperature with Tris-buffered saline $+0.1 \%$ Tween 20 (TBST) containing 5\% bovine serum albumin and incubated overnight with primary antibodies against
PPAR $\gamma$, Arg-1 and GAPDH (Cell Signaling Technology, Beverly, USA) at $4{ }^{\circ} \mathrm{C}$. The next day, membranes were incubated with secondary antibody (Cell Signaling Technology, Beverly, USA) for $1 \mathrm{~h}$ after three washes by TBST. The antigen-antibody complexes were detected using an enhanced chemiluminescence kit (Thermo Fisher Scientific, San Jose, USA).

\section{Statistical analysis}

Results were expressed as mean \pm standard deviation or median (interquartile range) as appropriate for continuous variables and as percentages for categorial variables. All data were analyzed using the SPSS 13.0 software (SPSS, Chicago, IL, USA). Two-tailed Student's non-paired $t$-test was applied for evaluating statistically significant differences between two independent groups. The correlation analysis was accessed by Pearson's correlation coefficient. Two-tailed $\mathrm{P}<0.05$ was considered to be statistically significant.

\section{Results}

\section{Clinical characteristics of AMI patients}

Among 35 AMI patients enrolled in the present study, the median age was 70 years (range, 39-87 years). Clinical characteristics were presented in Table 1. This group showed a higher proportion of patients with STEMI and multiple vessel involvement. The healthy control group consisted of 4 women and 21 men, with a median age of 70 years (range, 38-88 years) (Table $S 1)$.

\section{Circulating $\mathrm{CD}^{+} 8^{+} \mathrm{CD} 163^{+}$and $\mathrm{CX} 3 \mathrm{CR}^{+} \mathrm{CD} 163^{+} \mathrm{M} 2$ monocytes expanded and correlated positively in AMI}

The presence of $\mathrm{CD} 68^{+} \mathrm{CD} 163^{+}$and $\mathrm{CX} 3 \mathrm{CR} 1^{+} \mathrm{CD} 163^{+}$ M2 monocytes were explored in $\mathrm{HC}$ and AMI patients (Figure 1). $\mathrm{CD}_{68}{ }^{+} \mathrm{CD} 163^{+} \mathrm{M} 2$ monocytes were found significantly elevated in AMI patients as compared with $\mathrm{HC}$ $(20.68 \pm 1.40$ vs. $8.08 \pm 0.26, \mathrm{P}<0.01$, Figure $1 A, B)$. Similarly, the expansion of $\mathrm{CX} 3 \mathrm{CR} 1^{+} \mathrm{CD} 163^{+} \mathrm{M} 2$ monocytes were dramatically accentuated in AMI patients $(4.67 \pm 0.49 v s$. $0.58 \pm 0.08, \mathrm{P}<0.01$, Figure $1 A, C)$. A significant positive linear correlation between $\mathrm{CD} 68^{+} \mathrm{CD} 163^{+}$and $\mathrm{CX} 3 \mathrm{CR} 1^{+} \mathrm{CD} 163^{+}$ M2 monocytes was noted in AMI patients $(\mathrm{r}=0.39, \mathrm{P}=0.02$, Figure $1 E)$, but not among $\mathrm{HCs}(\mathrm{r}=0.09, \mathrm{P}=0.13$, Figure $1 D)$. These results furnished evidence that the percentage of $\mathrm{CX} 3 \mathrm{CR} 1^{+} \mathrm{CD} 163^{+} \mathrm{M} 2$ monocytes dramatically elevated and 
Table 1 Clinical characteristics of AMI patients

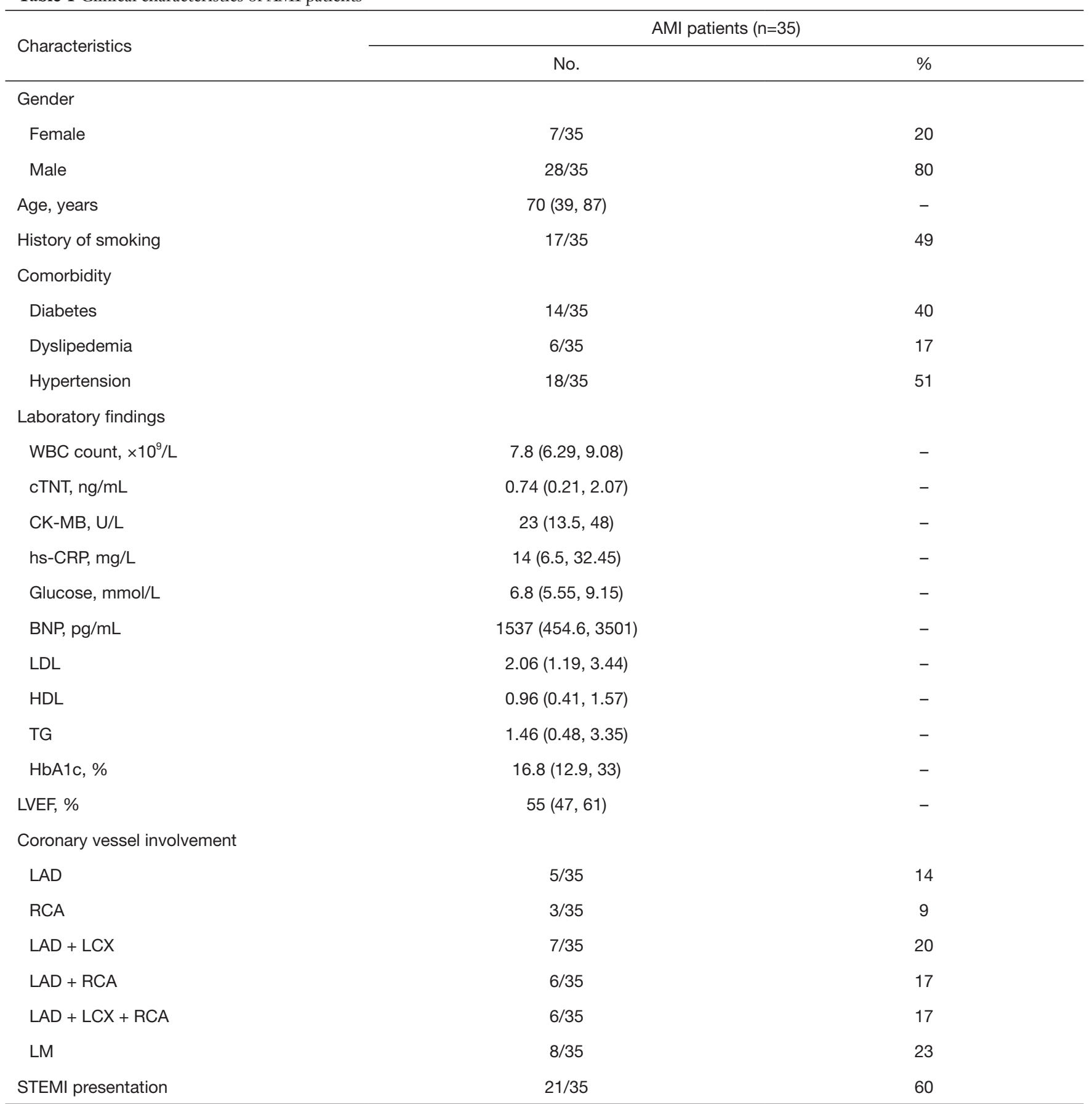

Data are presented as number (\%), median (interquartile range) of subjects. WBC, white blood cell; cTNT, troponin T; CK-MB, creatine kinase-MB; hsCRP, high sensitivity C-reactive protein; BNP, brain natriuretic peptide; LDL, low-density lipoprotein; HDL, high density lipoprotein; TG, triglyceride; HbA1c, hemoglobin A1c; LVEF, left ventricular ejection fraction; LAD, left anterior descending artery; LCX, left circumflex artery; LM, left main; RCA, right coronary artery; STEMI, ST segment elevation myocardial infarction. 

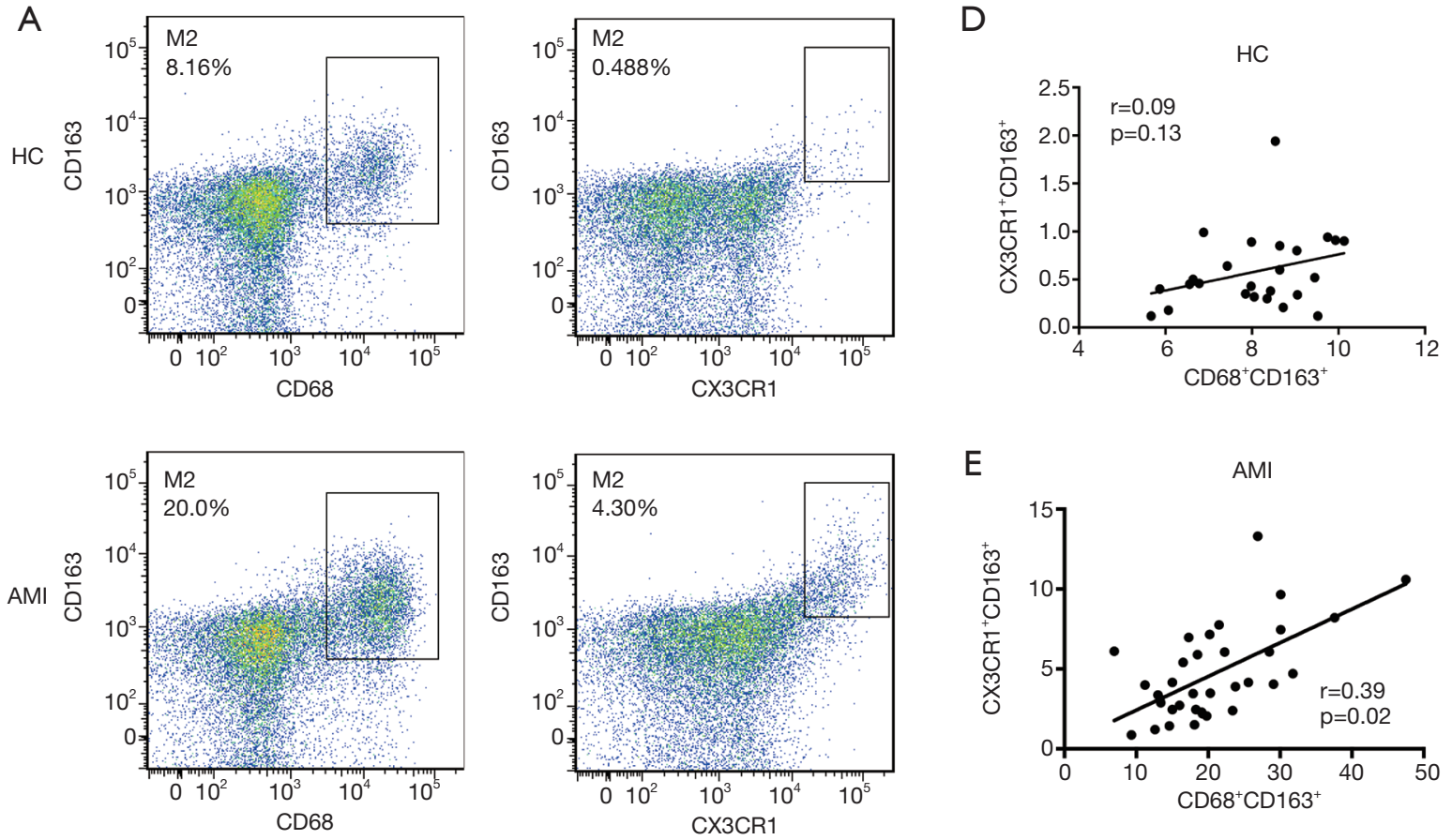

E

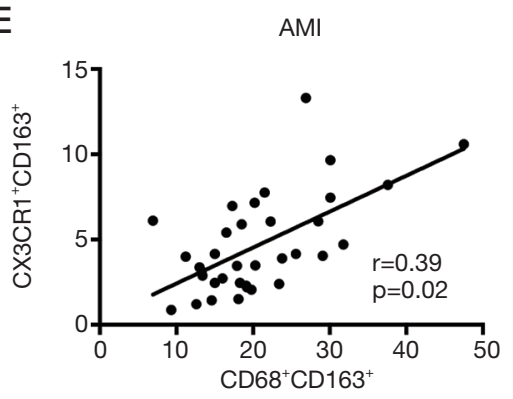

B

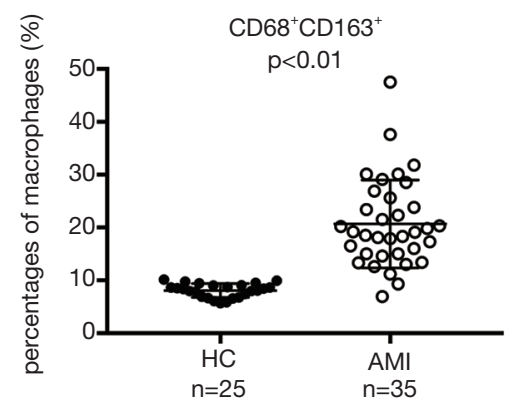

C

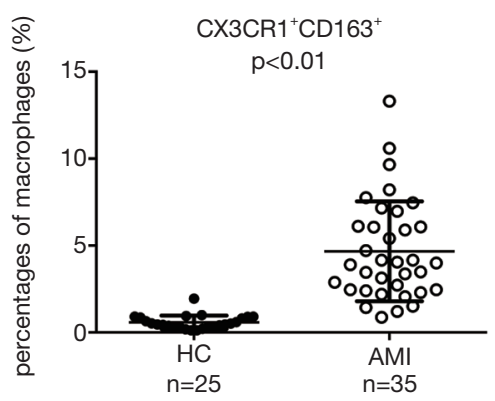

Figure 1 Circulating $\mathrm{CD} 68^{+} \mathrm{CD} 163^{+}$and $\mathrm{CX} 3 \mathrm{CR} 1^{+} \mathrm{CD} 163^{+} \mathrm{M} 2$ monocytes expanded and correlated positively in patients with $\mathrm{AMI}$. A Representative dot plots of $\mathrm{CD} 68^{+} \mathrm{CD} 163^{+}$and $\mathrm{CX} 3 \mathrm{CR} 1^{+} \mathrm{CD} 163^{+} \mathrm{M} 2$ monocytes in the PBMCs of $\mathrm{AMI}$ patients ( $\mathrm{n}=35$ ) and HCs ( $\mathrm{n}=25$ ). Peripheral $\mathrm{CD} 68^{+} \mathrm{CD} 163^{+}(\mathrm{B})$ and $\mathrm{CX} 3 \mathrm{CR} 1^{+} \mathrm{CD} 163^{+}(\mathrm{C}) \mathrm{M} 2$ monocytes were significantly elevated in AMI patients. Correlation between the percentage of $\mathrm{CD} 68^{+} \mathrm{CD} 163^{+}$and $\mathrm{CX} 3 \mathrm{CR} 1^{+} \mathrm{CD} 163^{+} \mathrm{M} 2$ monocytes in $\mathrm{HC}$ (D) and AMI patients (E). $\mathrm{P}<0.01$, compared to HCs in two-tailed Student's non-paired t test. Data were determined by Pearson's correlation test. AMI, acute myocardial infarction; HC, healthy control.

correlated positively with $\mathrm{CD} 68^{+} \mathrm{CD} 163^{+} \mathrm{M} 2$ monocytes in AMI patients.

\section{Cardiac specific biomarkers and acute-phase markers correlated positively with M2 monocytes}

Significant positive linear correlations were found between $\mathrm{CX} 3 \mathrm{CR} 1^{+} \mathrm{CD} 163^{+} \mathrm{M} 2$ monocytes and CK-MB ( $\mathrm{r}=0.54$, $\mathrm{P}<0.01$, Figure $2 A)$ and cTNT $(\mathrm{r}=0.63, \mathrm{P}<0.01$, Figure $2 A)$, while only cTNT was observed positively correlated with
$\mathrm{CD} 68^{+} \mathrm{CD} 163^{+} \mathrm{M} 2$ monocytes $(\mathrm{r}=0.37, \mathrm{P}=0.03$, Figure $2 B)$. In view of the notion that macrophages, hsCRP and glucose participate in acute-phase reaction, glucose $(\mathrm{r}=0.62, \mathrm{P}<0.01$, Figure $2 A)$ and hsCRP $(\mathrm{r}=0.58, \mathrm{P}<0.01$, Figure $2 A)$ were further tested and revealed that they were both positively correlated with $\mathrm{CX} 3 \mathrm{CR} 1^{+} \mathrm{CD} 163^{+} \mathrm{M} 2$ monocytes.

\section{CX3CR1 $1^{+}$monocytes had upregulated PPARy and Arg-1}

After magnetic cell separation of CX3CR $1^{+}$monocytes, 

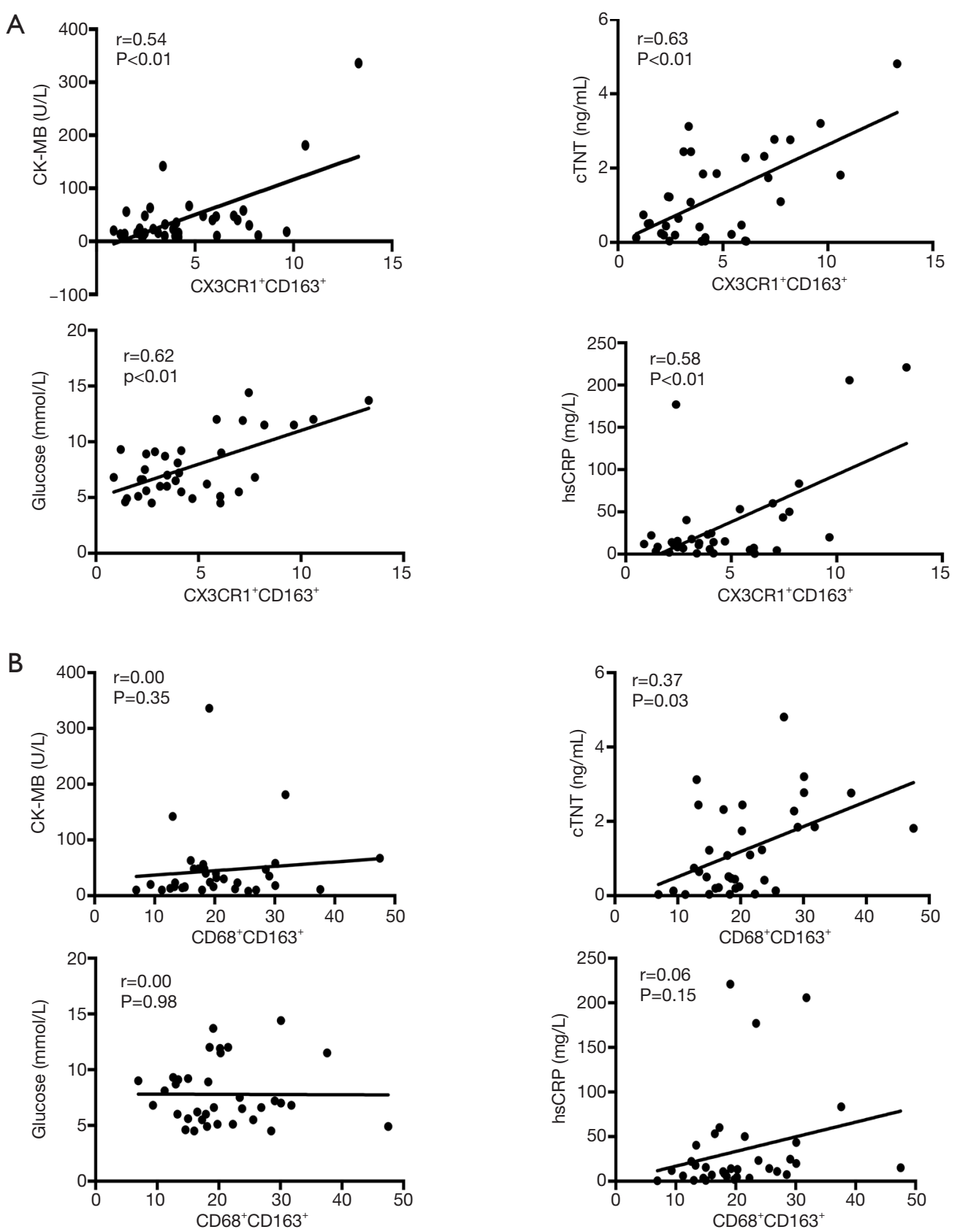

Figure 2 Cardiac specific biomarkers and acute-phase markers correlated positively with M2 monocytes. Correlation between cardiac specific biomarker (CK-MB and cTNT), acute-phase marker (glucose and hsCRP) and the percentages of circulating CX3CR1 ${ }^{+} \mathrm{CD} 163^{+}(\mathrm{A})$ and $\mathrm{CD}_{68}{ }^{+} \mathrm{CD} 163^{+}$(B) M2 monocytes in AMI patients $(\mathrm{n}=35)$. Data were determined by Pearson's correlation test.

the percentage of $\mathrm{CD} 68^{+} \mathrm{CD} 163^{+} \mathrm{M} 2$ monocytes was significantly elevated when compared with the whole PBMCs $(18.26 \pm 2.46$ vs. $48.33 \pm 2.16, \mathrm{P}<0.01$, Figure $3 A$ ). Thus, the $\mathrm{CX} 3 \mathrm{CR} 1^{+}$monocytes could be considered to share the phenotype of M2 macrophages. To further examine the function of $\mathrm{CX} 3 \mathrm{CR} 1^{+}$monocytes, we accessed the expression level of PPAR $\gamma$ and Arg- 1 in CX3CR1 ${ }^{+}$ monocytes. The relative quantitative mRNA level of PPAR $\gamma$ $(1.45 \pm 0.11$ vs. $1.09 \pm 0.07, \mathrm{P}=0.01)$ and Arg- $1(1.97 \pm 0.26$ vs. $1.09 \pm 0.08, \mathrm{P}<0.01)$ were significantly higher in $\mathrm{AMI}$ patients than those in $\mathrm{HC}$, the same was true for the expression of the corresponding protein (Figure 3B,C). 
A
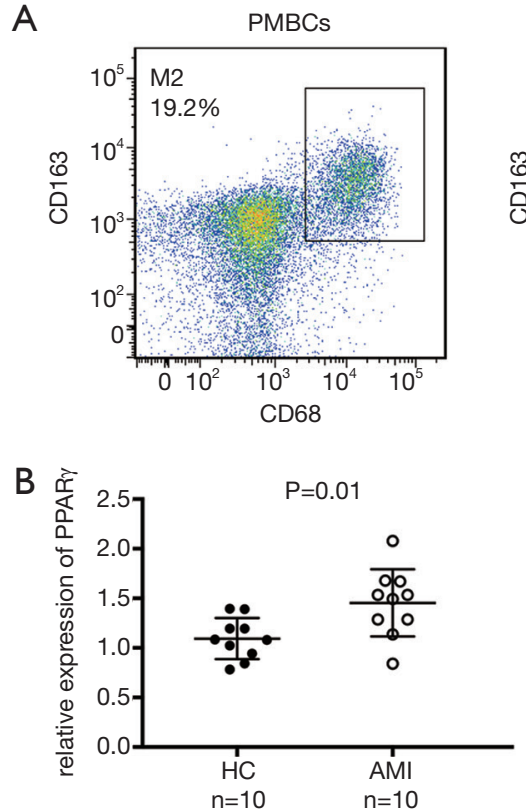
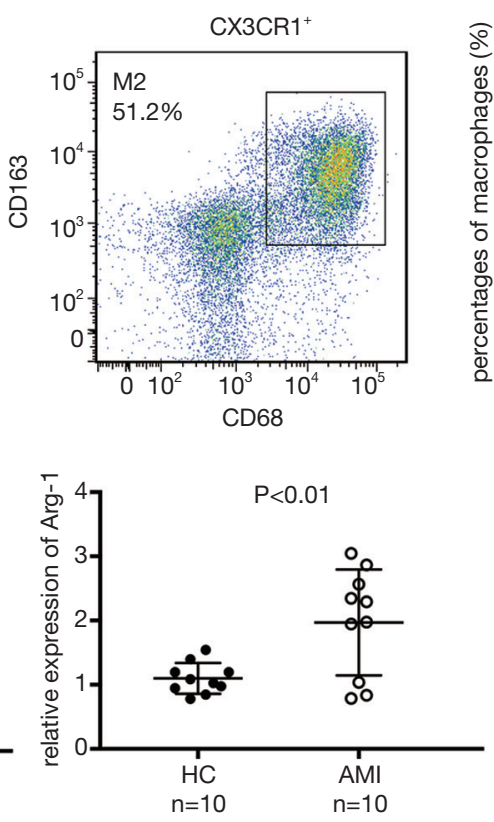

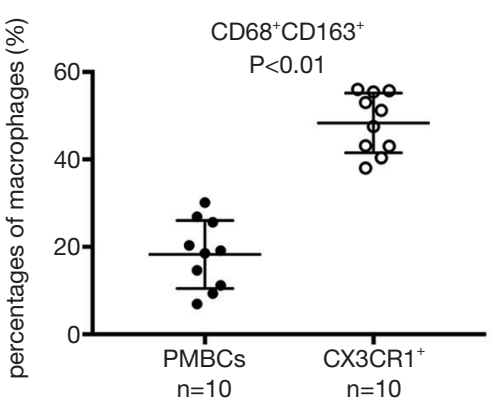

C

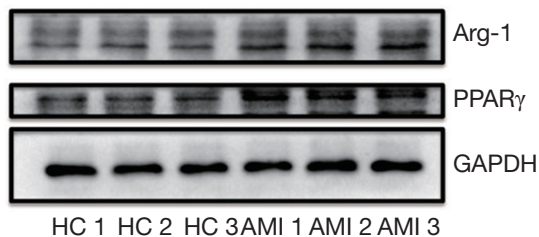

Figure 3 The upregulation of PPAR $\gamma$ and Arg-1 in CX3CR1+ monocytes. Representative dot plots of CD68 ${ }^{+} \mathrm{CD} 163^{+} \mathrm{M} 2-$ like macrophages in the PBMCs and CX3CR1 ${ }^{+}$monocytes of AMI patients (A). Real-time quantitative PCR analysis of the mRNA level (B) and Western blot analysis of the protein expression (C) of PPAR $\gamma$ and Arg- 1 in CX3CR1+ monocytes in HC $(n=10)$ and AMI patients $(n=10)$. P<0.01, compared to PBMCs in two-tailed Student's non-paired $t$ test. AMI, Acute myocardial infarction; HC, healthy control.

These results further validated these $\mathrm{CX} 3 \mathrm{CR} 1^{+}$monocytes to have features of polarized M2 macrophages.

\section{Discussion}

In the present study, the cell surface staining method (CX3CR1 and CD163) were used to identify the M2-like macrophages among circulating monocytes. The circulating $\mathrm{CX} 3 \mathrm{CR} 1^{+} \mathrm{CD} 163^{+} \mathrm{M} 2$ monocytes dramatically expanded and positively correlated with CK-MB, cTNT, hs-CRP and glucose among AMI patients. Moreover, the expression level of PPAR $\gamma$ and Arg- 1 were higher in $\mathrm{CX} 3 \mathrm{CR} 1^{+}$monocytes of AMI patients compared with those in HCs. These observations indicated that circulating $\mathrm{CX} 3 \mathrm{CR} 1^{+} \mathrm{CD} 163^{+}$ M2 monocytes might play a potential role in the early diagnosis of AMI.

Previous studies have highlighted that the increased total WBC count was positively associated with the adverse outcome in patients of ST segment elevation myocardial infarction and was predictive of higher six-month mortality in AMI $(20,21)$, however which leukocyte subtypes carry this risk is uncertain. We here found the extremely elevated circulating $\mathrm{CX} 3 \mathrm{CR} 1^{+} \mathrm{CD} 163^{+} \mathrm{M} 2$ monocytes among AMI patients. Unlike our previous intracellular staining methods (CD68 and CD163) (19), both CX3CR1 and CD163 expressed on the surface of monocytes, indicating that these cells exhibited more monocyte chemotactic ability. These findings sparked our great interest to speculate that the increased circulating $\mathrm{CX} 3 \mathrm{CR} 1^{+} \mathrm{CD} 163^{+} \mathrm{M} 2$ monocytes may be retained as the risk factor in $\mathrm{WBC}$ for cardiovascular risk prediction.

Intriguingly, we found the elevated circulating $\mathrm{CX} 3 \mathrm{CR} 1^{+} \mathrm{CD} 163^{+} \mathrm{M} 2$ monocytes positively correlated with the cardiac specific biomarkers, including cTNT and CK$\mathrm{MB}$, indicating that the circulating $\mathrm{CX} 3 \mathrm{CR} 1^{+} \mathrm{CD} 163^{+} \mathrm{M} 2$ monocytes might play a detrimental role in myocardial injury. The causes of plaque destabilization from an asymptomatic fibroatheroma plaque to high-risk unstable lesions remain largely unknown. Recent studies have revealed that intraplaque haemorrhages could accelerate the plaque destabilization, ultimately leading to acute cardiovascular events (8). Emerging data have indicated that the enhanced expression of CD163 positively correlated with intraplaque haemorrhages (22-24). Considering the significantly increased $\mathrm{CX} 3 \mathrm{CR} 1^{+} \mathrm{CD} 163^{+} \mathrm{M} 2$ monocytes in AMI, we would venture to speculate that they might 
contribute to intraplaque haemorrhage.

Many studies have clarified that progressive inflammation predisposes to plague rupture. CX3CR1 has been unveiled to augment the inflammatory via the crosstalk between smooth muscle cells and monocytes (25). The expression of CD163 in monocytes has been identified to be associated with intraplaque angiogenesis during the late inflammation (26). In agreement, the intriguing finding of the present study was the positive correlation between $\mathrm{CX} 3 \mathrm{CR} 1^{+} \mathrm{CD} 163^{+} \mathrm{M} 2$ monocytes and the typical acutephase marker, hs-CRP and glucose. Since inflammation and intraplaque angiogenesis often coexist at the base of advanced plaques, $\mathrm{CX} 3 \mathrm{CR} 1^{+} \mathrm{CD} 163^{+} \mathrm{M} 2$ monocytes might act as an inflammatory link between intraplaque angiogenesis and myocardial injury.

Notably, the cells we tested here were the populations circulating in the peripheral blood, not the polarized macrophages localized in the plaques. Although the tissueresident macrophages have been generally recognized as the main source of polarized-macrophages in myocardial tissue, circulating monocytes have been considered to be able to replenish the pool of tissue macrophages (27). Recently, a wealth of studies has existed on the role of monocyte-derived macrophages in plaque destabilization, shedding new light on the role of monocytes in intraplaque neoangiogenesis (28). $\mathrm{CD} 14^{+} \mathrm{CD} 16^{++}$cells, the non-classical monocytes, were found to patrol the vessel wall and expressing high level of CX3CR1 (29). The circulating $\mathrm{CX} 3 \mathrm{CR}^{+}$monocytes have been shown to be positively corelated with angiographic late lumen loss in AMI patients (30). Therefore, we speculated that the elevated circulating $\mathrm{CX} 3 \mathrm{CR} 1^{+} \mathrm{CD} 163^{+} \mathrm{M} 2$ monocytes might beget myocardial injury through patrolling the vessel and infiltrating in the plaque, and then differentiating into M2 macrophages.

Given the fact that circulating $\mathrm{CX} 3 \mathrm{CR} 1^{+} \mathrm{CD} 163^{+} \mathrm{M} 2$ monocytes positively correlated with cardiac specific and acute-phase markers in AMI, the CX3CR $1^{+}$monocytes were readily available, and the separated $\mathrm{CX} 3 \mathrm{CR} 1^{+}$monocytes expressed higher M2 specific marker (CD68 and CD163), we then analyzed the function of the CX3CR1 ${ }^{+}$monocytes to further our understanding this particular cell population. Despite functioned as a transcriptional regulator of genes linked to lipid metabolism, mounting evidence have suggested that PPAR $\gamma$ also plays an essential role in M2 macrophages polarization $(31,32)$. Also, the expression of Arg-1 has generally been recognized to precisely reflect the overall M2 polarization (18). It was intriguing to note that the expression level of both PPAR $\gamma$ and Arg- 1 in CX3CR1 $1^{+}$ monocytes of AMI patients were higher than those in HCs, indicating that $\mathrm{CX} 3 \mathrm{CR} 1^{+}$monocytes have the potential for polarized M2 macrophages. Finally, the circulating $\mathrm{CX} 3 \mathrm{CR} 1^{+} \mathrm{CD} 163^{+} \mathrm{M} 2$ monocytes might act as the valuable biomarker for plaque vulnerability in AMI.

\section{Limitations}

The finding in our study should be viewed in light of the limitation about the relatively small study population. It's still hard to claim an exact deduction to elucidate the contribution to angiogenesis of circulating $\mathrm{CX} 3 \mathrm{CR} 1^{+} \mathrm{CD} 163^{+} \mathrm{M} 2$ monocytes as we didn't examine the angiogenic growth factors and intraplaque haemorrhage in human coronary unstable plaques. Further tissuebased study and the cell function of the circulating CX3 CR $1^{+} \mathrm{CD} 163^{+} \mathrm{M} 2$ monocytes would be exciting to explore in AMI patients. More speculatively, these cells might be further applied to predict the risk of stable coronary heart disease.

\section{Conclusions}

Collectively, the increased circulating $\mathrm{CX} 3 \mathrm{CR} 1^{+} \mathrm{CD} 163^{+}$ M2 monocytes shared some vital characteristics of polarized M2 macrophages. Those positive correlation with cardiac specific biomarkers and acute-phase markers may open new avenues for their clinical application. Further studies will shed light on the prognostic value and more detailed molecular mechanism of the circulating $\mathrm{CX} 3 \mathrm{CR} 1^{+} \mathrm{CD} 163^{+}$ M2 monocytes in AMI.

\section{Acknowledgments}

Funding: This work was supported by grants from National Natural Science Foundation of China $(81870098,81600090$, 81500090, and 81470282), the Science and Technology Commission of Shanghai Municipality (18ZR1407200), and Shanghai Municipal Health Commission (201840351, and 20144Y0194). All authors obtain permission to acknowledge from all those mentioned in the acknowledgements.

\section{Footnote}

Data Sharing Statement: Available at http://dx.doi. 
org/10.21037/atm-20-383

Conflicts of Interest: All authors have completed the ICMJE uniform disclosure form (available at http://dx.doi. org/10.21037/atm-20-383). The authors have no conflicts of interest to declare.

Ethical Statement: The authors are accountable for all aspects of the work in ensuring that questions related to the accuracy or integrity of any part of the work are appropriately investigated and resolved. The study was in accordance with the ethical standards formulated in the Helsinki Declaration and was approved by the respective local Medical Ethics Committees. Written informed consent was obtained from each patient before being included in the study. All patient's personal data have been secured

Open Access Statement: This is an Open Access article distributed in accordance with the Creative Commons Attribution-NonCommercial-NoDerivs 4.0 International License (CC BY-NC-ND 4.0), which permits the noncommercial replication and distribution of the article with the strict proviso that no changes or edits are made and the original work is properly cited (including links to both the formal publication through the relevant DOI and the license). See: https://creativecommons.org/licenses/by-nc-nd/4.0/.

\section{References}

1. Reed GW, Rossi JE, Cannon CP. Acute myocardial infarction. Lancet 2017;389:197-210.

2. Thygesen K, Alpert JS, Jaffe AS, et al. Fourth universal definition of myocardial infarction (2018). Rev Esp Cardiol (Engl Ed) 2019;72:72.

3. Lu Y, Li L, Yan H, et al. Endothelial microparticles exert differential effects on functions of Th1 in patients with acute coronary syndrome. Int J Cardiol 2013;168:5396-404.

4. Ley K, Miller YI, Hedrick CC. Monocyte and macrophage dynamics during atherogenesis. Arterioscler Thromb Vasc Biol 2011;31:1506-16.

5. Tabas I, Bornfeldt KE. Macrophage Phenotype and Function in Different Stages of Atherosclerosis. Circ Res 2016;118:653-67.

6. Libby P, Tabas I, Fredman G, et al. Inflammation and its resolution as determinants of acute coronary syndromes. Circ Res 2014;114:1867-79.

7. Cho KY, Miyoshi H, Kuroda S, et al. The phenotype of infiltrating macrophages influences arteriosclerotic plaque vulnerability in the carotid artery. J Stroke Cerebrovasc Dis 2013;22:910-8.

8. de Vries MR, Quax PH. Plaque angiogenesis and its relation to inflammation and atherosclerotic plaque destabilization. Curr Opin Lipidol 2016;27:499-506.

9. Virmani R, Kolodgie FD, Burke AP, et al. Atherosclerotic plaque progression and vulnerability to rupture: angiogenesis as a source of intraplaque hemorrhage. Arterioscler Thromb Vasc Biol 2005;25:2054-61.

10. Sluimer JC, Kolodgie FD, Bijnens AP, et al. Thinwalled microvessels in human coronary atherosclerotic plaques show incomplete endothelial junctions relevance of compromised structural integrity for intraplaque microvascular leakage. J Am Coll Cardiol 2009;53:1517-27.

11. Parma L, Baganha F, Quax PHA, et al. Plaque angiogenesis and intraplaque hemorrhage in atherosclerosis. Eur J Pharmacol 2017;816:107-15.

12. Jetten N, Verbruggen S, Gijbels MJ, et al. Antiinflammatory $\mathrm{M} 2$, but not pro-inflammatory M1 macrophages promote angiogenesis in vivo. Angiogenesis 2014;17:109-18.

13. Corliss BA, Azimi MS, Munson JM, et al. Macrophages: An Inflammatory Link Between Angiogenesis and Lymphangiogenesis. Microcirculation 2016;23:95-121.

14. Fukui S, Iwamoto N, Takatani A, et al. M1 and M2 Monocytes in Rheumatoid Arthritis: A Contribution of Imbalance of M1/M2 Monocytes to Osteoclastogenesis. Front Immunol 2018;8:1958.

15. Fadini GP, de Kreutzenberg SV, Boscaro E, et al. An unbalanced monocyte polarisation in peripheral blood and bone marrow of patients with type 2 diabetes has an impact on microangiopathy. Diabetologia 2013;56:1856-66.

16. Pucci S, Mazzarelli P, Zonetti MJ, et al. CX3CR1 receptor polymorphisms, Th1 cell recruitment, and acute myocardial infarction outcome: looking for a link. Biomed Res Int 2013;2013:451349.

17. Bouhlel MA, Derudas B, Rigamonti E, et al. PPARgamma activation primes human monocytes into alternative M2 macrophages with anti-inflammatory properties. Cell Metab 2007;6:137-43.

18. Wynn TA, Barron L. Macrophages: master regulators of inflammation and fibrosis. Semin Liver Dis 2010;30:245-57.

19. Shao $X, W u B$, Cheng $L$, et al. Distinct alterations of CD68(+)CD163(+) M2-like macrophages and myeloidderived suppressor cells in newly diagnosed primary 
immune thrombocytopenia with or without CR after highdose dexamethasone treatment. J Transl Med 2018;16:48.

20. Sabatine MS, Morrow DA, Cannon CP, et al. Relationship between baseline white blood cell count and degree of coronary artery disease and mortality in patients with acute coronary syndromes: a TACTICS-TIMI 18 (Treat Angina with Aggrastat and determine Cost of Therapy with an Invasive or Conservative Strategy- Thrombolysis in Myocardial Infarction 18 trial)substudy. J Am Coll Cardiol 2002;40:1761-8.

21. Barron HV, Harr SD, Radford MJ, et al. The association between white blood cell count and acute myocardial infarction mortality in patients $>$ or $=65$ years of age: findings from the cooperative cardiovascular project. J Am Coll Cardiol 2001;38:1654-61.

22. Chen F, Eriksson P, Kimura T, et al. Apoptosis and angiogenesis are induced in the unstable coronary atherosclerotic plaque. Coron Artery Dis 2005;16:191-7.

23. Moreno JA, Ortega-Gomez A, Delbosc S, et al. In vitro and in vivo evidence for the role of elastase shedding of CD163 in human atherothrombosis. Eur Heart J 2012;33:252-63.

24. Bentzon JF, Otsuka F, Virmani R, et al. Mechanisms of plaque formation and rupture. Circ Res 2014;114:1852-66.

25. Butoi ED, Gan AM, Manduteanu I, et al. Cross talk between smooth muscle cells and monocytes/activated monocytes via CX3CL1/CX3CR1 axis augments expression of pro-atherogenic molecules. Biochim Biophys Acta 2011;1813:2026-35.

Cite this article as: Shao $\mathrm{X}, \mathrm{Wu} \mathrm{B}$, Chen $\mathrm{P}$, Hua F, Cheng L, Li F, Zhan Y, Liu C, Ji L, Min Z, Sun L, Cheng Y, Chen H. Circulating CX3CR1+CD163+ M2 monocytes markedly elevated and correlated with cardiac markers in patients with acute myocardial infarction. Ann Transl Med 2020;8(9):578. doi: 10.21037/atm-20-383
26. Buechler C, Ritter M, Orso E, et al. Regulation of scavenger receptor CD163 expression in human monocytes and macrophages by pro- and antiinflammatory stimuli. J Leukoc Biol 2000;67:97-103.

27. Yang J, Zhang L, Yu C, et al. Monocyte and macrophage differentiation: circulation inflammatory monocyte as biomarker for inflammatory diseases. Biomark Res 2014;2:1.

28. Melter M, Reinders ME, Sho M, et al. Ligation of CD40 induces the expression of vascular endothelial growth factor by endothelial cells and monocytes and promotes angiogenesis in vivo. Blood 2000;96:3801-8.

29. Gostner JM, Fuchs D. Biomarkers for the role of macrophages in the development and progression of atherosclerosis. Atherosclerosis 2016;255:117-8.

30. Liu Y, Imanishi T, Ikejima H, et al. Association between circulating monocyte subsets and in-stent restenosis after coronary stent implantation in patients with ST-elevation myocardial infarction. Circ J 2010;74:2585-91.

31. Zhang T, Shao B, Liu GA. Rosuvastatin promotes the differentiation of peripheral blood monocytes into M2 macrophages in patients with atherosclerosis by activating PPAR-gamma. Eur Rev Med Pharmacol Sci 2017;21:4464-71.

32. Yao Q, Liu J, Zhang Z, et al. Peroxisome proliferatoractivated receptor gamma (PPARgamma) induces the gene expression of integrin alphaVbeta5 to promote macrophage M2 polarization. J Biol Chem 2018;293:16572-82. 

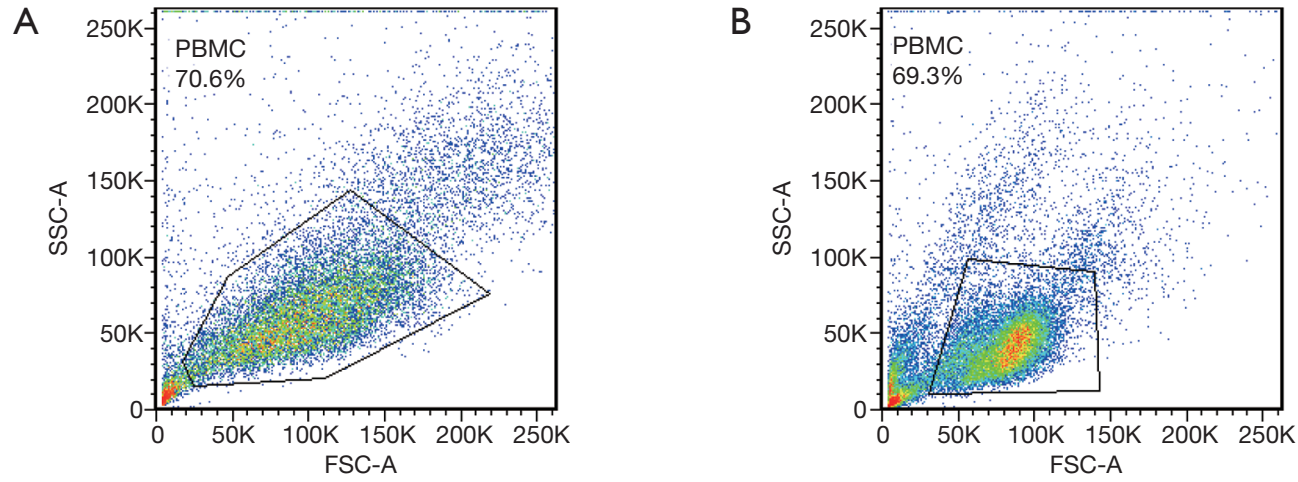

Figure S1 The FSC $\times$ SSC gate of PBMCs for gating $\mathrm{CD} 68^{+} \mathrm{CD}_{163^{+}}(\mathrm{A})$ and $\mathrm{CX} 3 \mathrm{CR} 1^{+} \mathrm{CD} 163^{+}(\mathrm{B}) \mathrm{M} 2$ monocytes.

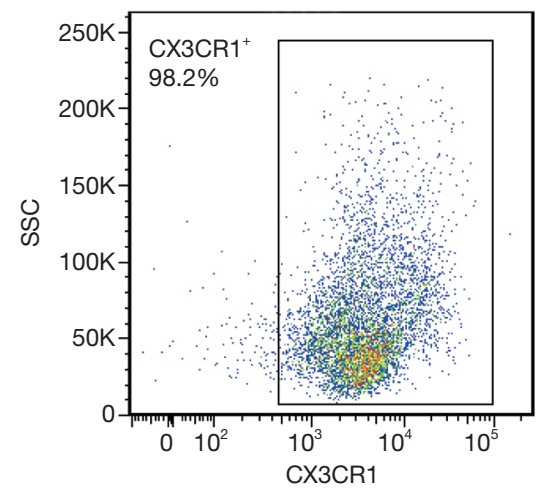

Figure S2 The purity of CX3CR1 $1^{+}$monocytes tested by FCM. 
Table S1 Clinical characteristics of healthy controls

\begin{tabular}{|c|c|c|}
\hline Characteristics & \multicolumn{2}{|c|}{ Healthy controls $(n=25)$} \\
\hline \multicolumn{3}{|l|}{ Gender } \\
\hline Female & $4 / 25$ & 16 \\
\hline Male & $21 / 25$ & 84 \\
\hline History of smoking & $11 / 25$ & 44 \\
\hline \multicolumn{3}{|l|}{ Comorbidity } \\
\hline Diabetes & $0 / 25$ & 0 \\
\hline Dyslipedemia & $5 / 25$ & 20 \\
\hline WBC count, $\times 10^{9} / \mathrm{L}$ & $6.95(3.99,9.2)$ & - \\
\hline cTNT, ng/mL & $0.018(0.003,0.029)$ & - \\
\hline CK-MB, U/L & $14(7,23)$ & - \\
\hline hs-CRP, mg/L & $1.5(0.3,3.1)$ & - \\
\hline Glucose, $\mathrm{mmol} / \mathrm{L}$ & $4.6(3.5,5)$ & - \\
\hline BNP, pg/mL & $105(46,246)$ & - \\
\hline LDL & $2.14(1.19,3.98)$ & - \\
\hline HDL & $1.57(1.02,3.42)$ & - \\
\hline
\end{tabular}

Data are presented as number (\%), median (interquartile range) of subjects. WBC, white blood cell; cTNT, troponin T; CK-MB, creatine kinase-MB; hsCRP, high sensitivity C-reactive protein; BNP, brain natriuretic peptide; LDL, low-density lipoprotein; HDL, high density lipoprotein; TG, triglyceride; HbA1c, hemoglobin A1c. 